\title{
Tratamiento quirúrgico y reconstrucción del cáncer periorbitario
}

Periocular skin cancer's surgical treatment and reconstruction

\section{Miguel Olmos ${ }^{1}$, Nataly Portilla ${ }^{2}$, Carlos Castro ${ }^{3}$.}

1. Médico dermatólogo, cirujano dermatólogo, especialista en Cirugía de Mohs, Servicio de Dermatología, Hospital de San José, Fundación Universitaria de Ciencias de la Salud, Bogotá, D.C., Colombia.

2. Médica y cirujana; coordinadora médica, Clínica Erasmo, Valledupar, Colombia.

3. Médico epidemiólogo; instructor asociado, División de Investigaciones, Fundación Universitaria de Ciencias de la Salud, Bogotá, D.C., Colombia.

\section{Resumen}

Introducción. Se ha estimado que, aproximadamente, 5 a $10 \%$ de todos los cánceres de piel se ubican en la región periorbitaria. El párpado y, en general, la región periorbitaria son sitios anatómicos asociados a un gran riesgo de recurrencia y metástasis. Las recomendaciones sugieren practicar cirugía micrográfica de Mohs y escisión de segmentos por congelamiento.

Objetivo. Describir los antecedentes y el contexto actual del tratamiento quirúrgico y la reconstrucción para cáncer periorbital.

Métodos. Se hizo una búsqueda en la literatura científica, utilizando las palabras clave (MeSH): neoplasms, carcinoma, squamous cell, basosquamous, melanoma, Mohs surgery, y operadores booleanos. Las bases de datos donde se hizo la búsqueda fueron: Pubmed, Ebsco Host, Embase, Lilacs, Science direct y Ovid.

Conclusiones. La cirugía micrográfica de Mohs es actualmente la opción terapéutica y diagnóstica más completa y con menores tasas de recidiva para el tratamiento quirúrgico de tumores cutáneos complejos; además, permite ahorrar tejido sano con la seguridad de la erradicación tumoral. Se sugiere realizar estudios analíticos para establecer una mejor evidencia.

PALABRAS CLAVE: neoplasias, carcinoma, escamocelular, basocelular, melanoma, cirugía de Mohs.

\section{Summary}

Introduction. It is estimated that approximately 5 to $10 \%$ skin cancers are located at the periocular area. Eyelids and the periorbital region are anatomic sites associated with high recurrence and metastasis risk. The literature suggests to realize Mohs micrographic surgery or the frozen section technique.

Objective. To describe the periorbital cancer surgical treatment and reconstruction background and the recent context.

\section{Correspondencia:}

Miguel Olmos

Email:

miolmos@hotmail.com

Recibido: 24 de febrero de 2013.

Aceptado: 10 de junio de 2013.

No se reportan conflictos de intereses. 
Methods: We made a literature search using the following MeSH terms: Neoplasms, Carcinoma, Squamous Cell, Basosquamous, Melanoma, Mohs surgery and Boolean operators. We performed the search in Pubmed, Ebsco host, Embase, Lilacs, Science direct and Ovid databases.

Conclusions: Mohs micrographic surgery is nowadays the most complete therapeutic and diagnostic method and it presents the lowest recurrence rate for complex skin tumor surgical treatment, besides it permits to save tumorfree tissue with secure margins. We suggest carrying-out more analytic studies to establish better evidence.

KEY WORDS: Neoplasms, carcinoma, squamous Cell, basosquamous, melanoma, Mohs surgery.

\section{Introducción}

El cáncer de piel localizado en la región periorbitaria es infrecuente ${ }^{1,2}$. Se ha estimado que, aproximadamente, 5 a $10 \%$ de todos los cánceres de piel se ubican en la región periorbitaria, con mayor frecuencia en mayores de 60 años y con una relación entre hombres y mujeres de 3 a 13.4. El párpado y, en general, la región periorbitaria son sitios anatómicos asociados a un gran riesgo de recurrencia y metástasis. Dado el riesgo de invasión de la órbita, la nasofaringe y el cráneo, se vuelve imperiosa la necesidad de obtener márgenes totalmente libres de tumor $^{5,6}$. Las recomendaciones basadas en la 'evidencia' sugieren únicamente dos modalidades terapéuticas para estos tumores cutáneos de la región periorbitaria, las cuales se caracterizan por el control permanente de sus bordes: la cirugía micrográfica de Mohs y la escisión de segmentos por congelamiento, ya que proporcionan un mayor control de los márgenes, disminuyendo las recidivas tumorales ${ }^{7-15}$.

\section{Métodos de búsqueda}

\section{Estrategia}

Se hizo una búsqueda sistemática de la literatura científica con las siguientes palabras clave (MeSH): neoplasms, carcinoma, squamous cell, basosquamous, melanoma y Mohs surgery, las cuales se combinaron utilizando operadores booleanos AND, OR y NOT; se limitó la búsqueda por año de publicación (desde el 2000 hasta la fecha) y tipo de diseño (revisiones, series de casos, estudios descriptivos, analíticos y experimentales). No se tuvo en cuenta el idioma de la publicación.
Las bases de datos donde se hicieron las búsquedas fueron: Pubmed, Ebsco Host, Embase, Lilacs, Science Direct y Ovid. Se encontraron y evaluaron 224 artículos, de los cuales, se seleccionaron 48 , que fueron la fuente bibliográfica para este artículo. Además, se hizo una búsqueda de literatura gris y fuentes primarias, como libros y entrevistas a profesionales de la comunidad académica, experimentados en diagnóstico y tratamiento quirúrgico de cáncer periorbitario.

El objetivo fue describir los antecedentes y el contexto actual del tratamiento quirúrgico y la reconstrucción para cáncer periorbital.

\section{Clasificación}

\section{Carcinoma basocelular}

Es el tipo tumoral más frecuente en la región periorbitaria y corresponde a $90 \%$ de los tumores malignos palpebrales. El $54 \%$ de este tipo histológico se presenta en el párpado inferior, debido a la mayor exposición solar. En segundo lugar, se presenta en el canto interno, localización que, ligada a su origen embriológico, empeora su pronóstico pues facilita la invasión de estructuras más profundas, como el conducto lacrimal, la órbita posterior, los senos paranasales, la fosa craneal anterior, la nasofaringe y el periostio $0^{3,16-19}$.

\section{Carcinoma escamocelular}

En la región periocular, el carcinoma escamocelular ocupa el segundo lugar en frecuencia y corresponde a 5 a $10 \%$ de los tumores periorbitarios; le siguen el melanoma, el carcinoma sebáceo y el carcinoma microquístico de los anexos, los cuales son relativamente 
infrecuentes. Al igual que el carcinoma basocelular, el escamocelular es más frecuente en el párpado inferior que en el superior, con una razón de 4 a 1, y ocupa el tercer lugar en frecuencia en el canto interno $0^{3,4,20}$.

\section{Melanoma}

Ocupa el tercer lugar en orden de frecuencia entre las lesiones malignas de piel. El más frecuente en la región periorbitaria es el lentigo maligno, que suele localizarse en el párpado inferior. El melanoma se caracteriza por su amplia extensión subclínica, lo cual conlleva altas tasas de recurrencia después de la escisión estándar.

Existen controversias en cuanto a las modalidades terapéuticas para la resección del melanoma, debido a la dificultad para diferenciar las células melanóticas malignas de los melanocitos atípicos, los cuales se localizan en el margen del tumor y pueden ser secundarios a daño solar ${ }^{3,4}$. Sin embargo, la información disponible favorece la cirugía micrográfica de Mohs para el tratamiento del melanoma in situ o de poca profundidad (menos de $1 \mathrm{~mm}$ ), en la región periorbitaria. Daniel, et al., consideran como la mejor opción terapéutica cirugía de Mohs modificada o lenta, debido a sus menores tasas de recurrencia local y las ventajas en ahorro de tejido sano, en comparación con la cirugía de Mohs clásica; esta técnica tiene una dificultad y es el tiempo diferido, ya que si se realiza por congelación, como se hace con la técnica clásica, puede originar artefactos por el frío, que hacen más difícil la evaluación de los melanocitos. Los partidarios de la técnica tradicional de Mohs, la defienden debido a la posibilidad de reconstruir en forma inmediata el defecto quirúrgico ${ }^{8,10}$.

\section{Tumores cutáneos de baja incidencia en la región periorbitaria}

Además del carcinoma basocelular, el carcinoma escamocelular y de los melanomas, otros tumores menos frecuentes que también pueden presentarse son el carcinoma sebáceo, el carcinoma microquístico de los anexos, también conocido como carcinoma esclerosante del conducto sudoríparo, y el carcinoma de células de Merkel o carcinoma neuroendocrino.

A diferencia de los primeros mencionados, el carcinoma sebáceo se presenta con mayor frecuencia en el párpado superior y tiene una alta tasa de metástasis, que alcanza hasta el $30 \%$, lo cual hace indispensable su resección completa para evitar recurrencias locales y a distancia ${ }^{4,20}$. El carcinoma microquístico de los anexos ocurre típicamente en cabeza y cuello, con mayor frecuencia en los labios, es infrecuente en la región periorbitaria y corresponde al $12 \%$ de los periorbitarios. Los pocos casos reportados de este tumor, se localizaron principalmente en el párpado inferior, seguido del canto interno, las cuales son zonas de mayor exposición solar. Debido a su alto riesgo de invasión perineural y de recidiva con la escisión local amplia, la cirugía micrográfica de Mohs o la escisión de segmentos por congelamiento, se han convertido en el tratamiento estándar del carcinoma microquístico de los anexos s,9,12,21. $^{2}$

El carcinoma de células de Merkel es un tumor cutáneo maligno que raramente aparece en región periorbitaria; sin embargo, es de los más agresivos. Las guías actuales de la National Comprehensive Cancer Network, avalan como tratamientos la escisión local completa con márgenes de al menos $2 \mathrm{~cm}$, la cirugía de Mohs modificada o la cirugía de Mohs clásica ${ }^{4,9,22-24}$.

\section{Técnicas terapéuticas}

\section{Cirugía micrográfica de Mohs}

La cirugía micrográfica de Mohs asegura un margen totalmente libre de tumor y provee al cirujano mayor confianza para la planeación de una reconstrucción del defecto quirúrgico ${ }^{8,9,13}$. Esta técnica fue descrita por Fredrick E. Mohs en 1936, y se basa en la extracción del tumor por escisión quirúrgica.

Se toma una porción delgada (aproximadamente, 2 $\mathrm{mm}$ ) de toda la superficie peritumoral para el análisis por secciones de piel congelada. Posteriormente, se aplana, se orienta y se marca, para permitir que la parte exterior se corte horizontalmente después de la congelación, lo cual garantiza que todos los bordes peritumorales y el lecho sean visibles en un solo plano.

Después de la verificación histológica, si no hay más focos tumorales, los márgenes se consideran libres de enfermedad. De lo contrario, el tumor residual está marcado para permitir la escisión posterior, que se lleva a cabo en paralelo a la zona de la cual se eliminó el tejido. Esta escisión en rebanadas sucesivas requiere una estrecha coordinación entre la cirugía, la preparación y el análisis histológico; sin embargo, incrementa el tiempo quirúrgico e implica más personal, el cual debe tener entrenamiento, lo que aumenta los costos de tratamiento ${ }^{13,14,25}$.

La reconstrucción se hace, en la mayoría de los casos, de forma inmediata después de la resección ${ }^{24} \mathrm{y}$ el análisis total del tumor. Con otros métodos quirúrgicos de resección tumoral, como la cirugía micrográfica de Mohs con inclusión en un bloque de parafina (o cirugía de Mohs lenta) o enfoques similares (Breuningery), los resultados histológicos no están disponibles de inmediato, ya que el tejido se incluye en parafina. La cirugía de Mohs sigue 
siendo un procedimiento complejo que implica el estudio histopatológico inmediato de la pieza quirúrgica ${ }^{14}$.

En sus publicaciones de 1986, Mohs documentó tasas de curación a cinco años de $99 \%$ para el carcinoma basocelular y de 98,1\% para el escamocelular, usando la cirugía micrográfica para tumores del párpado. Sin embargo, a lo largo de la historia de la medicina se han ofrecido múltiples opciones para el tratamiento de los tumores cutáneos de la región periorbitaria, como la criocirugía, la escisión estándar con márgenes y fijación permanente, la radioterapia o medicamentos tópicos como el imiquimod ${ }^{4,13,19,20}$.

Aunque en un principio Mohs planteó la granulación del defecto quirúrgico como una buena opción para la cicatrización, en 1986 Anderson planteó que este método podría generar problemas funcionales y estéticos de la región periocular, por lo que sugirió una reconstrucción quirúrgica de los defectos producidos en esta área por las resecciones mediante cirugía micrográfica de Mohs ${ }^{4,9,13}$.

\section{Técnicas de reconstrucción}

Aunque hay muchas causas de deformidad en la región periorbitaria, como colobomas, traumas y retracciones cicatriciales, entre otros, la resección de las neoplasias malignas de piel, aún representan la principal causa de reconstrucción de la región periocular ${ }^{3}$. Pese a que no hay consenso respecto al mejor método para la reconstrucción de los defectos periorbitarios después de la resección tumoral, contamos con los diferentes colgajos descritos en la literatura científica, que han dado buenos resultados según lo demostrado por estudios experimentales, dependiendo de la complejidad de la deformidad, la localización, el tamaño, la edad del pa-

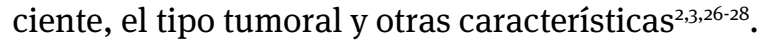

Un colgajo de piel se diferencia de un injerto porque el primero, además de la piel, involucra el tejido subcutáneo con su plexo subdérmico de vasos (pedículo), por lo cual tiene su propio suministro sanguíneo, sin importar el lugar donde sea ubicado ${ }^{3,14}$. Los injertos se clasifican según su espesor. Tanto los delgados como los de espesor medio, solo pueden obtenerse con un dermátomo ${ }^{3}$.

Para la adecuada adhesión de los injertos, hay ciertos aspectos que siempre deben tenerse en cuenta:

1. Hemostasia del lecho receptor.

2. Sutura cuidadosa de los bordes.

3. Cuidados posteriores a la reconstrucción, como la aplicación de un rollo de gaza compresivo por al menos cuatro días (capitoneo).

4. Superficie del injerto mayor de un tercio del área por cubrir, especialmente en casos de retracción cicatricial.
5. Los colgajos bipediculados disminuyen el riesgo de necrosis, ya que tiene un suministro sanguíneo de patrón de doble azar 5 .

La reconstrucción de grandes defectos periorbitarios (mayores de $3 \mathrm{~cm}$ ) constituye un gran desafío para los cirujanos, por lo que se requiere personal perfectamente capacitado y entrenado en la fisiología de la cicatrización, la anatomía facial y los principios reconstructivos de las distintas técnicas de colgajos e injertos de tejidos blandos, desde el diseño hasta el traslado y colocación del colgajo.

La aplicación de todo lo anterior debe verse reflejada en reconstrucciones con resultados estéticos y funcionales adecuados ${ }^{1,3,13,24,29}$. Antes de la reconstrucción del defecto quirúrgico, el cirujano debe asegurarse de tener márgenes libres de tumor, lo cual puede lograrse en el mismo tiempo quirúrgico en el caso de la cirugía de Mohs, o puede diferirse en los casos en que se utilizan otras técnicas de resección del tumor, como la cirugía de Mohs lenta. Sin embargo, cuando sea posible, debe procurarse una reconstrucción no diferida, puesto que disminuye el riesgo de infección y retracción de estructuras, ya que no ha empezado el proceso de granulación $\mathrm{n}^{1,3,13,24,28,30,31}$.

Si no se cuenta con la cirugía de Mohs, se puede hacer un estudio histopatológico de los márgenes del tumor y la reconstrucción inmediata es una buena opción en los siguientes casos:

1. Si no se trata de un tumor recurrente.

2. Si el tumor no es infiltrante, no es un tipo histológico de alto riesgo y los bordes están bien delimitados.

3. Si se tiene un margen amplio (de, al menos, $4 \mathrm{~mm}$ ).

4. Si se trata de un paciente mayor, con gran riesgo anestésico.

En casos graves de tumores con extensión ósea, debe hacerse, además, una osteotomía durante la resección tumoral, pero, si el compromiso es superficial, la intervención puede consistir en la ablación del periostio y el raspado del hueso solamente. Se cuenta con la opción de injertos óseos, que deben utilizarse sólo cuando se requiera recubrir una estructura subyacente importante, como la duramadre; lo ideal es evitarlos. En defectos muy extensos de la pared orbitaria, un buen recurso son los injertos aloplásticos. Algo que no debe olvidarse es no dejar bordes óseos afilados, para evitar la penetración de injertos y colgajos en los tejidos blandos subyacentes ${ }^{1,13,29}$.

Los injertos deben obtenerse de sitios adyacentes al defecto, pues garantizan mejores resultados estéticos por la semejanza de color y textura con la piel resecada, y aunque se prefieren de espesor completo, cuando no 
hay suficiente piel donante disponible, se puede optar por injertos de espesor parcial. Los sitios donantes de elección son: el párpado superior, la zona preauricular, la retroauricular, la supraclavicular y la región interna del brazo ${ }^{1,28-31}$.

Por el alto grado de irrigación de la cara, el plexo vascular subdérmico suele suplir las necesidades para la integración de cualquier injerto, por lo que rara vez se requieren colgajos rotacionales. Sin embargo, hay ciertos factores, como la historia previa de cirugías faciales, el tabaquismo, la radiación o la enfermedad vascular, que pueden disminuir la circulación sanguínea y dificultar la adhesión del injerto, los cuales deben tenerse en cuenta ${ }^{1,27,29}$. Para obtener colgajos de avance de tejido blando y minimizar la tensión del colgajo de avance, pueden hacerse incisiones paralelas a las líneas de tensión de la piel relajada. El hecho de que el cáncer de piel se presente con mayor frecuencia en pacientes mayores, presenta una ventaja quirúrgica al proporcionar una mayor capacidad de extensión y elasticidad de la piel ${ }^{1,29,30}$.

Cuando se avanzan colgajos, para evitar la tensión excesiva en la piel, deben usarse suturas de soporte más profundas sobre la capa del sistema músculo-aponeurótico superficial, que simulen la función del ligamento órbito-malar, sobre todo en defectos centro-faciales. El afrontamiento de la piel debe hacerse libre de tensión, con bordes evertidos, usando tantos puntos de colchonero como sean necesarios ${ }^{1,14,30,31}$.

Las técnicas reconstructivas básicas pueden consistir en cierre directo, colgajos o injertos; y en defectos de mayor tamaño, se pueden utilizar varias técnicas a la vez. Pueden clasificarse según el área anatómica que requiere el tratamiento: párpado superior, párpado inferior, canto interno o canto externo. Lo ideal es reconstruir por subunidades anatómicas ${ }^{3,14}$.

\section{Párpado inferior}

En la reconstrucción del párpado inferior, el injerto debe fijarse en su aspecto más superior para evitar la tensión vertical inferior y los vectores negativos ${ }^{1,33^{1-34}}$.

Colgajo de Mustardé: es el método más conocido para la reconstrucción del párpado inferior, debido a que es una técnica simple y que da buenos resultados, tanto en defectos parciales como en defectos totales del párpado inferior, ya que proporciona un adecuado suministro sanguíneo que garantiza la supervivencia del colgajo, por la similitud en color y textura de la piel, lo cual da un excelente resultado estético y disimula adecuadamente la cicatriz, y porque puede practicarse en un solo tiempo quirúrgico.

Consiste en hacer un amplio colgajo de avance y rota- ción de la mejilla, que se diseña de manera triangular y se traslada hasta el defecto del párpado inferior; algunas veces se puede asociar a un injerto condromucoso del tabique nasal. Sin embargo, es una cirugía bastante amplia que algunas veces requiere anestesia general y que, como secuela, puede dejar ectropión, epífora y afectación de la rama frontal del nervio facial. Para evitar el ectropión se han propuesto las suturas de suspensión, las cuales se fijan desde el tarso hasta la región frontal, evitando la retracción durante el proceso de adhesión y cicatrización. Se retiran cuando se ha terminado la integración del colgajo a los tejidos adyacentes, entre la primera y segunda semana después

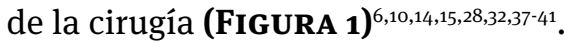

Colgajo de Tenzel: es un colgajo de avance semicircular, excelente opción en la reparación de defectos de espesor completo del párpado, que pueden ser centrales, mediales o laterales. Se utiliza cuando está comprometida entre 33 y $75 \%$ de la longitud horizontal del párpado inferior, o de 30 a $60 \%$ de la longitud horizontal del párpado superior.

Aunque es una técnica muy similar a la de Mustardé, provee una reconstrucción funcionalmente más eficiente de las lamelas del párpado, con mejor reorganización tisular ${ }^{14}$ Se intervienen ambas lamelas, anterior y posterior, en un único tiempo quirúrgico. Se prefiere esta técnica cuando se dispone de segmentos del párpado adyacentes a ambos lados del defecto, de espesor completo, que sean laxos y tengan disponibilidad de piel del canto lateral. También, es una mejor opción que los injertos cuando hay antecedentes de irradiación o quemadura pues, como es un colgajo, provee su propia irrigación sanguínea (FIGURA 2) ${ }^{14,40}$.

Se debe practicar una cantotomía lateral (línea arqueada semicircular superior de $20 \mathrm{~mm}$, aproximadamente) debajo del colgajo y se secciona la rama inferior del tendón cantal lateral, para movilizar completamente el segmento inferior lateral del párpado. El colgajo se diseca totalmente y se avanza medialmente, de tal forma que el tejido lateral del párpado se avanza hasta el borde medial del defecto. Posteriormente, debe hacerse una fijación cantal lateral, lo cual evita la caída del párpado. Las complicaciones del colgajo de Tenzel incluyen escotaduras del párpado, ectropión, pliegues cantales laterales, deformidades en trap-door y simbléfaron (FIGURA 2) ${ }^{14}$.

Colgajo tarso-conjuntival de Hughes: es un colgajo de tarso autógeno irrigado y revestido con la conjuntiva, de elección para defectos de espesor completo que impliquen entre 66 y $100 \%$ de la extensión horizontal del párpado inferior, que proporciona un reemplazo ideal de la lamela posterior. Las mejores opciones para la reconstrucción de la lámina posterior del párpado son los 

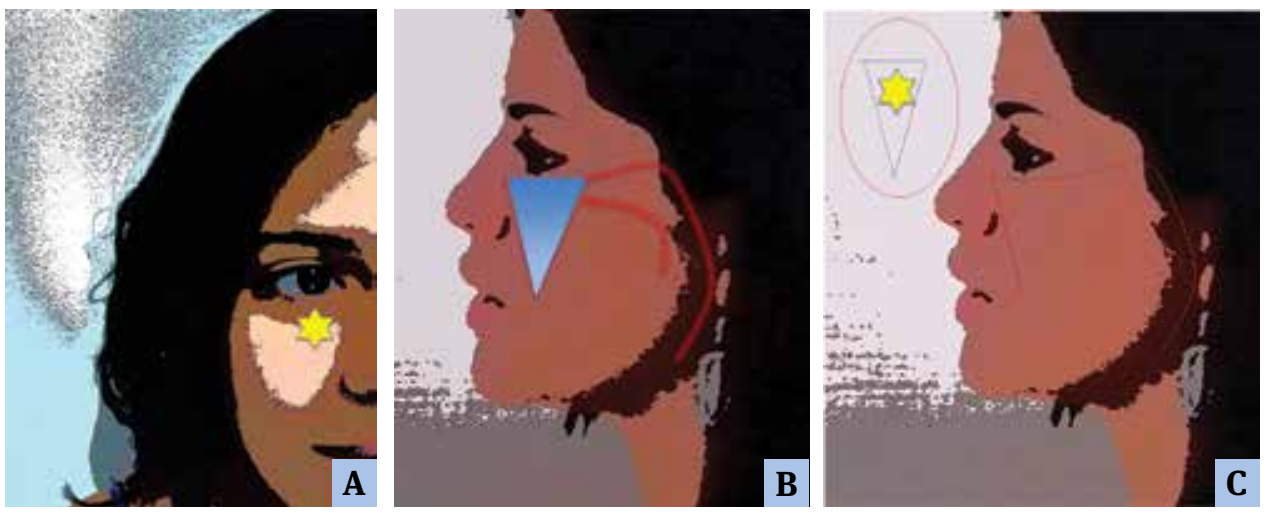

ESQUEMA DEL COLGAJO DE MUSTARDÉ.

A. Lesión tumoral.

B. El defecto se diseña de forma triangular y se avanza el colgajo.

c. Cierre definitivo.
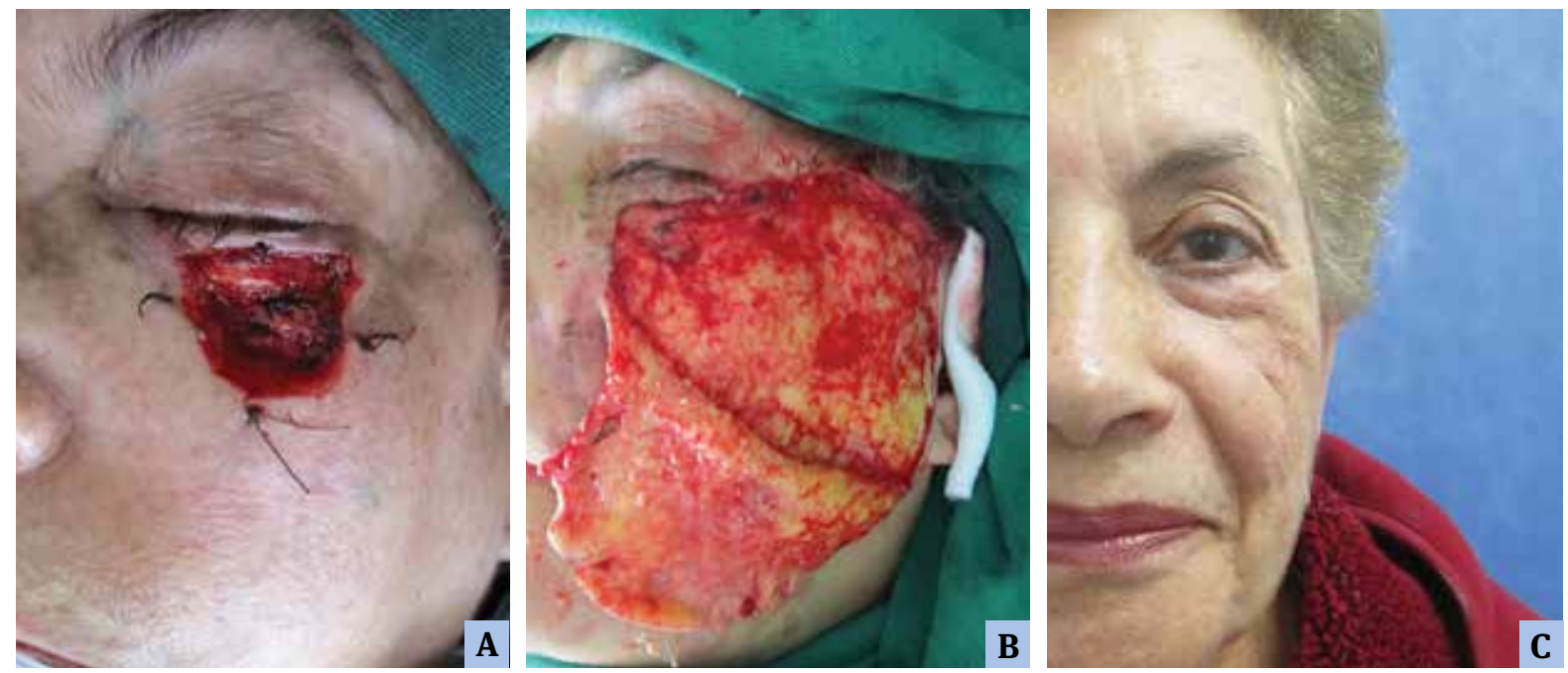

FIGURA 1. A. Primer tiempo de la cirugía micrográfica de Mohs -variación de parafina- en el tratamiento de un melanoma lentigo maligno. B. Se diseca el colgajo de Mustardé mediante el sistema músculo-aponeurótico superficial. C. Resultado dos meses después.

colgajos con pedículo o los colgajos libres del párpado opuesto. Sin embargo, cuando no se cuenta con suficiente tejido tarsal, los colgajos autólogos son la mejor alternativa. Otras opciones viables, pero menos frecuentes, son los injertos de mucosa del paladar duro (de elección), de cartílago auricular y de cartílago nasal ${ }^{42}$. Cuando hay gran pérdida de volumen, la lamela posterior puede reemplazarse con la superficie dérmica de los colgajos de dermis profunda ${ }^{14}$.

Para la reconstrucción de la lamela anterior, hay varias opciones, como un injerto cutáneo de espesor completo o un colgajo de avance local. Todos ellos deben hacerse respetando las unidades estéticas, para lograr el mejor resultado estético y funcional posible, puesto que evitan la tensión vertical (gravitacional) de la piel (particularmente en el párpado inferior) y reducen el riesgo de retracción del párpado o la mejilla y el ectropión. La lámina anterior (piel y músculo orbicular), debe suplir el aporte sanguíneo en casos de colgajos libres de lámina posterior y, viceversa, en casos de colgajos libres de la lámina anterior ${ }^{1,14}$.

La obstrucción visual temporal, hasta que el colgajo sea dividido en una segunda etapa de 7 a 45 días después del primer procedimiento, es un efecto secundario de este procedimiento ${ }^{14,17}$.

\section{Párpado superior}

Una adecuada reconstrucción y los cuidados posoperatorios son primordiales en la reparación del párpado superior, ya que este constituye la principal fuente de protección de la córnea ${ }^{1,3,14,28,30,31,37}$. Para la reconstrucción del párpado superior se han descrito las mismas técnicas reconstructivas que en el párpado inferior, aunque también describimos otra técnica quirúrgica usada para defectos de mayor tamaño ${ }^{29}$.

Colgajo de Cutler Beard: es la mejor opción para la reconstrucción de defectos mayores del párpado su- 

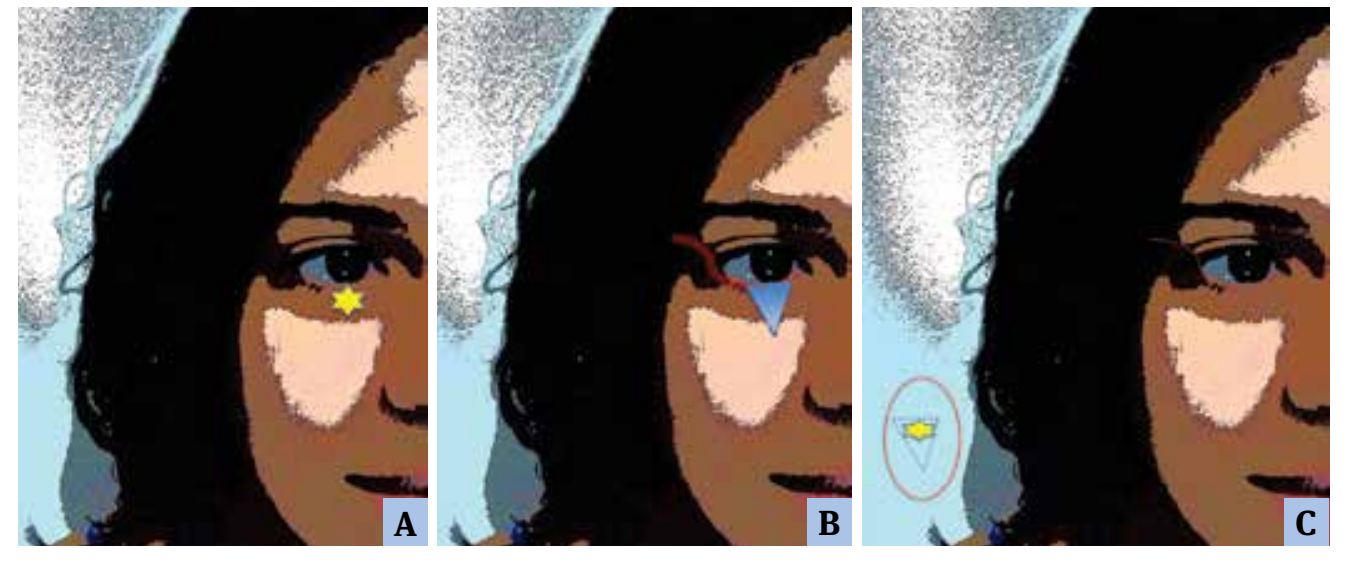

ESQUEMA DEL COLGAJO DE TENZEL.

SU BIOMECÁNICA ES LA MISMA DEL COLGAJO DE MUSTARDÉ.

A. Lesión tumoral.

B. Diseño de una línea arqueada semicircular superior.

C. Cierre inmediato; no se debe olvidar fijar el ligamento cantal externo.
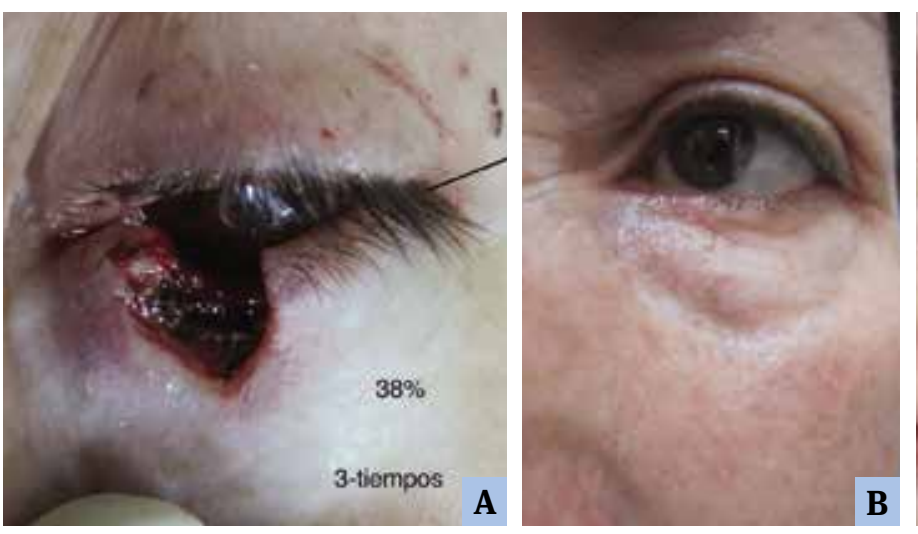

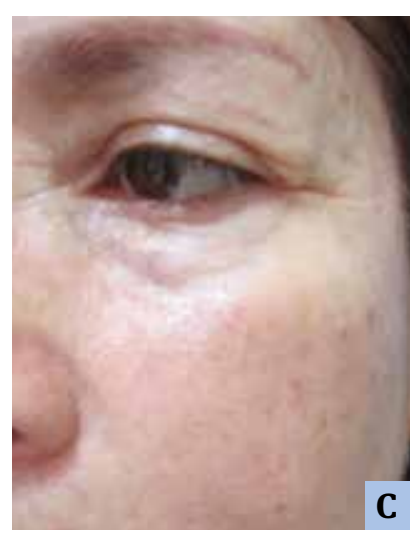

\section{FIGURA 2.}

A. Posoperatorio inmediato de la cirugía micrográfica de Mohs en defecto del $38 \%$ del párpado (colgajo de Tenzel).

B. Cuatro meses después de la cirugía.

C. Ocho meses después de la cirugía. perior y consiste en un colgajo de piel, músculo y conjuntiva, que se avanza desde el párpado inferior hasta el párpado superior, por lo cual el ojo queda cerrado al menos cuatro a seis semanas, hasta que se separa para la evaluación corneal. Dado que no es posible la reconstrucción de la lamela posterior mediante esta técnica quirúrgica, la inestabilidad del margen palpebral es uno de los efectos secundarios frecuentes de esta cirugía ${ }^{14}$.

Colgajo de doble avance en la zona supraciliar: se trata de colgajos unipediculados bilaterales, técnica también conocida como cierre en $\mathrm{H}$ o $\mathrm{H}$ plastia. Consiste en practicar dos incisiones opuestas al defecto, con lo cual se forman dos colgajos que se aproximan entre sí para cubrir el defecto, formando una H. No necesariamente los colgajos tienen que ser del mismo tamaño.

Esta técnica se puede utilizar en defectos paramediales de la zona frontal y la ciliar (FIGURA 4). La ventaja de esta técnica sobre los colgajos simples, es que distribuye su tensión desde los dos lados evitando, en el cierre, la desviación del de la línea media. En ocasiones se pueden utilizar los triángulos de Burow para lograr un mayor desplazamiento del colgajo. Por lo general, estos son cuatro y se colocan al final del colgajo para lograr un camuflaje de la cicatriz.

\section{Canto interno}

Es de suma importancia asegurar un anclaje rígido del canto, que provea una adecuada tensión horizontal, para lograr mantener la integridad, estabilidad, posición y función de los párpados y, así, evitar la exposición de la córnea (FIGURA 5) ${ }^{1,29,31,43}$.

Lindgren, et al., recomiendan usar un tubo de silicona para reemplazar el canalículo cuando se comprometa durante la resección tumoral. También, refieren el uso de técnicas combinadas de granulación de parte de la herida, más colgajos de espesor completo para la reconstrucción del canto interno ${ }^{13}$. Un injerto perióstico es una excelente opción en la reconstrucción del canto interno, para fijar la lamela posterior del párpado (tarso y conjuntiva), recrear los ligamentos del canto o soportar un colgajo superpuesto. Si no se cuenta con el periostio, porque se ha removido durante la resección tumoral, 

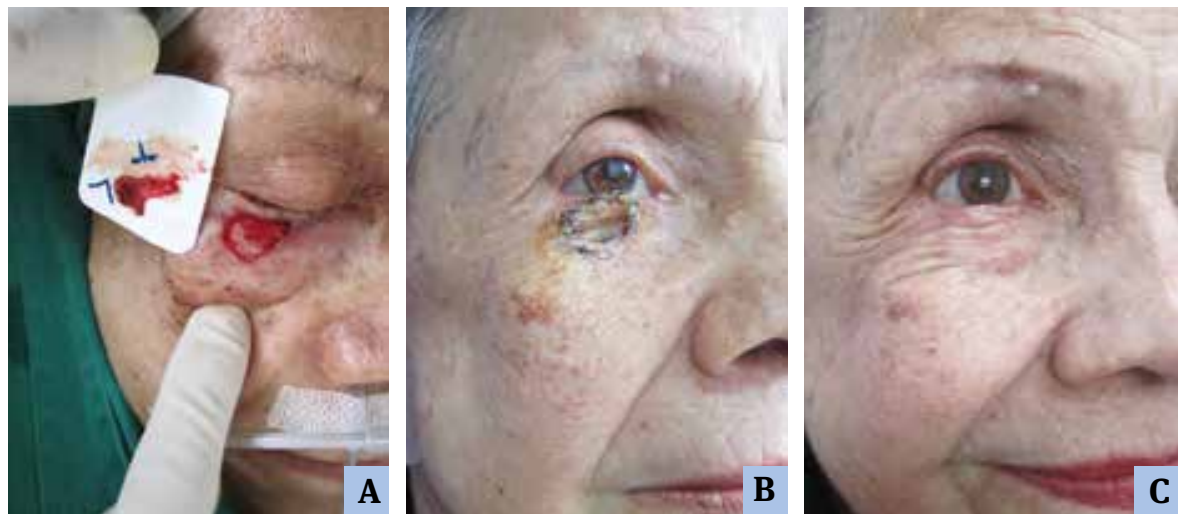

\section{FIGURA 3.}

A. Segundo tiempo de la cirugía micrográfica de Mohs en el tratamiento de un carcinoma basocelular trabecular.

B . Injerto preauricular 15 días después de la cirugía. C. Control a los cuatro meses.
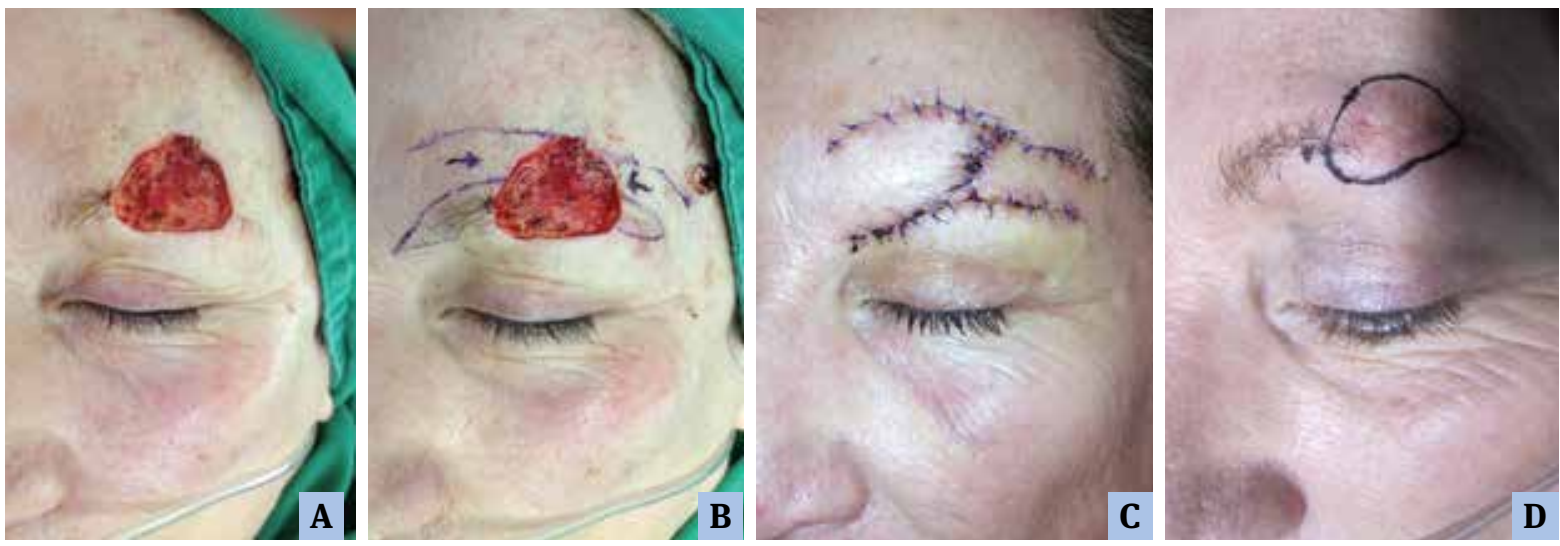

FIGURA 4. A. Placa tumoral de márgenes moderadamente definidos. B. Segundo tiempo de la cirugía micrográfica de Mohs. C. Diseño del colgajo de doble avance, incluyendo la ceja. D. Resultado quince días después de la cirugía.

puede optarse por una pequeña placa de titanio o un dispositivo de anclaje, fijados con sutura ${ }^{1,29,30,43}$.

Dos excelentes opciones para la reconstrucción del canto interno son los injertos cutáneos y los colgajos de Rigel ${ }^{44}$.

Injertos de piel: son una opción frecuentemente utilizada. Cuando se eligen como técnica reconstructiva, la mayor similitud en el color la ofrece un injerto de la región preauricular (FIGURAS 3, 5 Y 6). En la región periorbitaria, no deberían usarse injertos de espesor parcial alrededor de los párpados, pues facilitan las contracciones del injerto, llevando a ectropión y retracción del párpado inferior, de la mejilla o de ambos ${ }^{5,34}$.

Colgajo de Rigel: es un colgajo de transposición y rotación tomado de la línea medial frontal, también llamado colgajo glabelar, que debe trasladarse para cubrir el defecto del canto interno. Una posible secuela que debe tenerse en cuenta para la reconstrucción del canto interno, es el crecimiento de cejas en esta región si el colgajo es tomado de muy arriba ${ }^{15,45}$.

\section{Canto externo}

El canto lateral es una estructura compleja e importante de la región periorbitaria, no solo porque define la posición y función del párpado, su movimiento y tono muscular en reposo, sino porque también ayuda a determinar la apariencia racial y étnica de la fisura palpebral (FIGURA 6). Por ende, los daños del área cantal externa pueden dar como resultado cierre palpebral incompleto, exposición de la córnea, epífora y problemas estéticos ${ }^{46}$. Por esto, aunque en las últimas décadas se han mantenido los principios básicos de la reconstrucción del canto lateral, como la estabilización de rutina de esta estructura, las técnicas quirúrgicas han cambiado $5,34,47-50$.

Para las intervenciones quirúrgicas de esta área debe tenerse amplio conocimiento del recorrido de las ramas del nervio facial, para evitar daños accidentales de estas estructuras, lo cual podría resultar en déficits funcionales y estéticos ${ }^{5,48,51}$. Dependiendo de la extensión del 

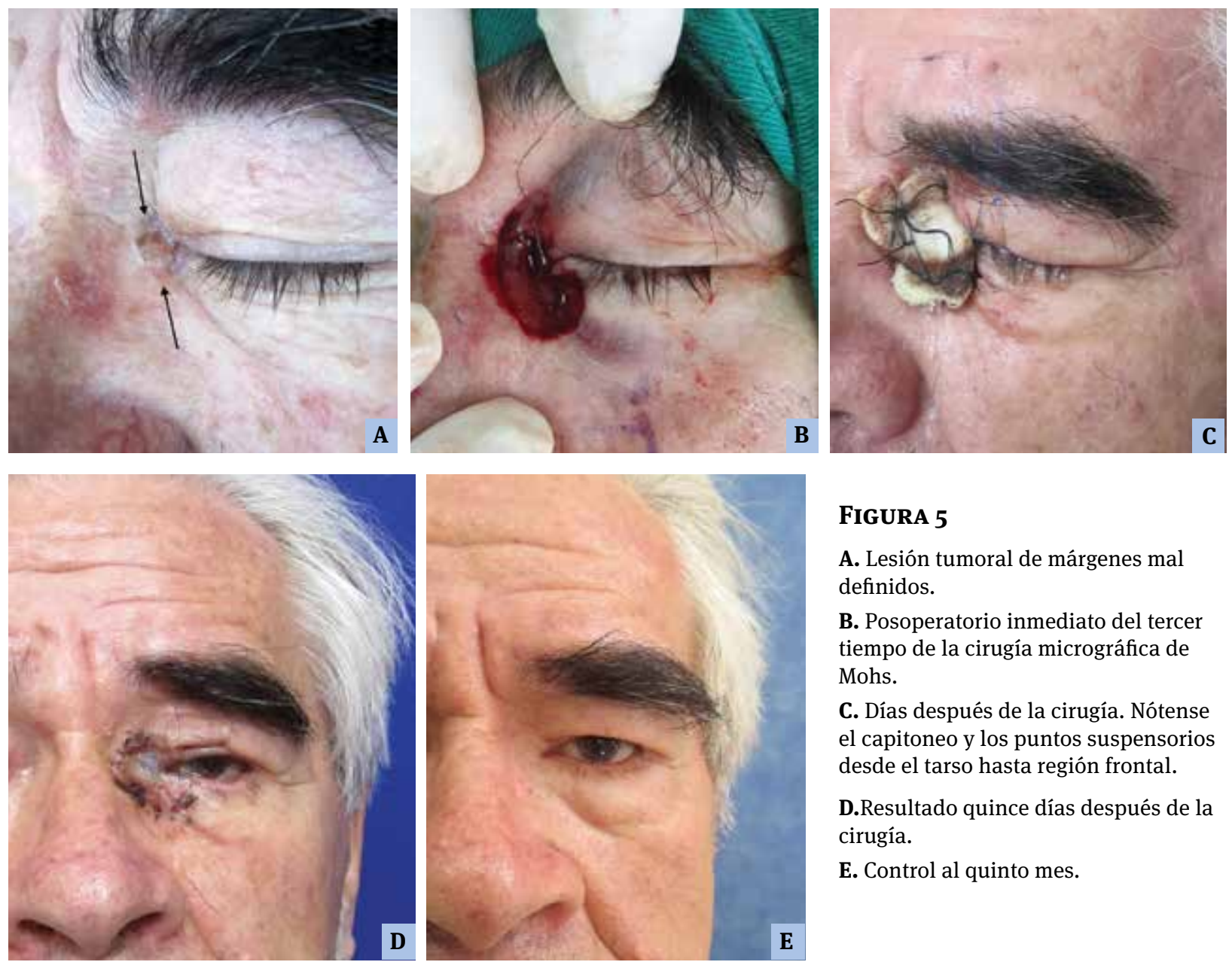

\section{FIGURA 5}

A. Lesión tumoral de márgenes mal definidos.

B. Posoperatorio inmediato del tercer tiempo de la cirugía micrográfica de Mohs.

C. Días después de la cirugía. Nótense el capitoneo y los puntos suspensorios desde el tarso hasta región frontal.

D.Resultado quince días después de la cirugía.

E. Control al quinto mes.

defecto y su complejidad, se debe escoger la técnica de reconstrucción más adecuada, entre las cuales podemos contar con las siguientes:

Cierre primario: es el método de reconstrucción más adecuado, simple y con menos complicaciones en casos de pequeños defectos. Se recomienda suturar el defecto en la dirección de las líneas de tensión sobre la piel relajada, pues esto disminuye el riesgo de dehiscencia y permite mejores resultados estéticos. Sin embargo, por la disposición en "pata de gallo" de las arrugas en el canto lateral, las cuales no siempre siguen las líneas de expresión, debe tenerse cuidado para orientar apropiadamente las cicatrices sobre el valle natural que se extiende horizontalmente entre una arruga de esta "pata de gallo" 5 . Además, debido a la poca disposición de dermis con que se cuenta en esta región, debe intentarse un cierre de piel libre de tensión, para evitar la dehiscencia de la herida. También, debe tenerse precaución en el momento de orientar la sutura, para no mover el canto lateralmente, la línea de implantación del pelo medialmente, ni la ceja inferiormente ${ }^{34,50,51}$.

Colgajo romboidal: descritos por Limberg, los colgajos romboideos se recomiendan en la reconstrucción del canto lateral.

Esta técnica consiste en la escisión del defecto en forma de rombo, ubicando los ángulos de $120^{\circ}$ en las áreas de mayor exceso de piel. Después, desde el vértice del ángulo de $120^{\circ}$, se traza una línea hacia afuera, de igual longitud a un lado del rombo, y se practica un corte a $60^{\circ}$ en el extremo de esta línea. Esta línea debe ser paralela y de igual longitud a un lado del rombo.

El colgajo se secciona y se traslada de tal forma que el corte del vértice de $60^{\circ}$ se inserte en el ángulo de $60^{\circ}$ del defecto romboideo. Este colgajo produce deformidades mínimas en las estructuras anatómicas adyacentes y, además, provee una piel similar para la reconstrucción del defecto ${ }^{5,47,49}$.

Colgajos de Fricke: es un colgajo en el que se hace 

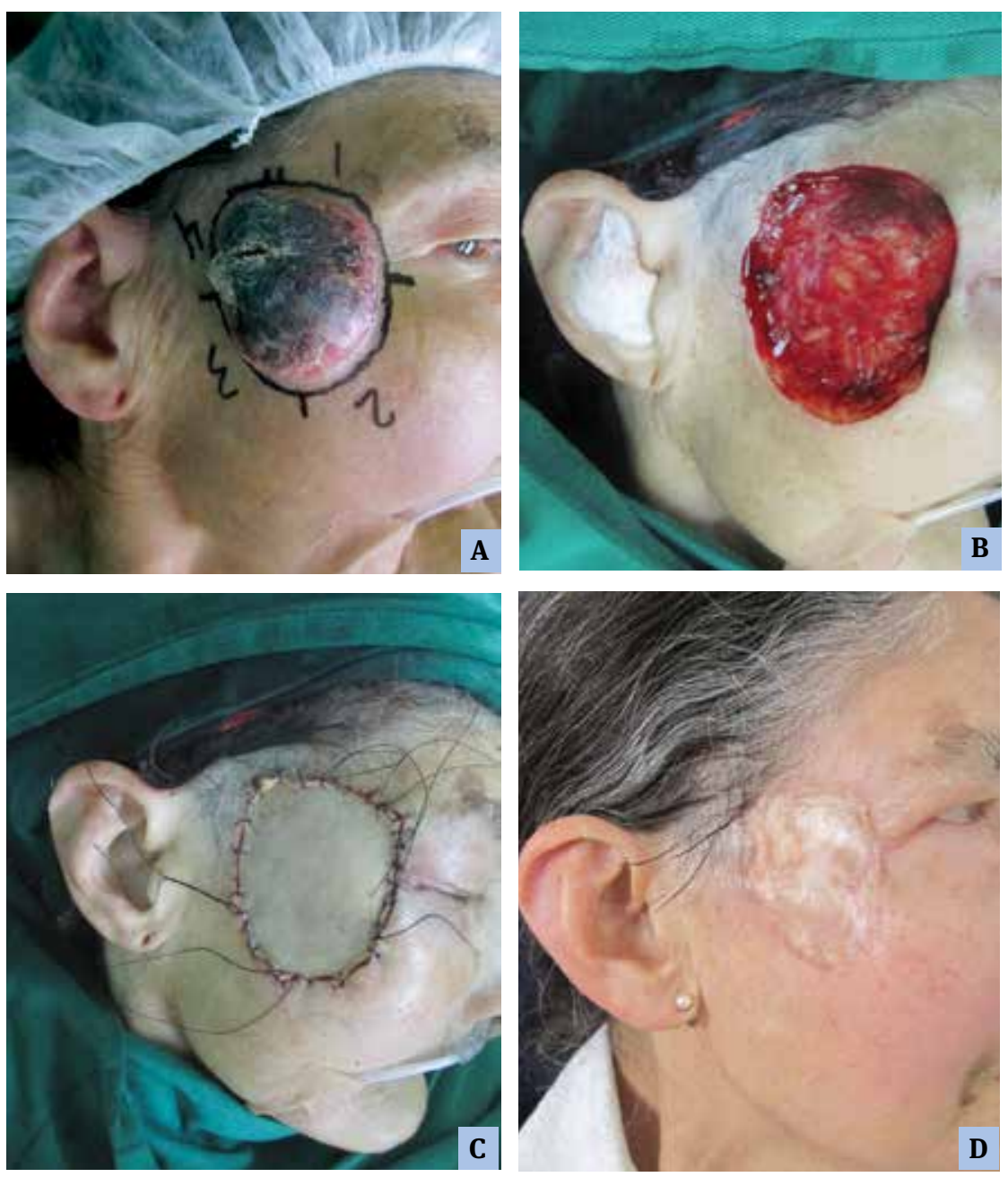

FIGURA 6. A.Carcinoma escamocelular de diferenciación queratósica. B . Tres tiempos de la cirugía micrográfica de Mohs. C. Colocación de injerto abdominal. Nótense el capitoneo y los puntos suspensorios desde el tarso hasta región frontal. D.Resultado un mes después de la cirugía. transposición de su base inferior. Se diseña en la piel de la región frontal o temporal y se rota medialmente para cubrir defectos del canto lateral, teniendo la precaución de no mover la ceja lateralmente o la línea de implantación del cabello anteriormente. Los colgajos grandes de este tipo pueden requerir sutura del sitio donante, lo cual puede dejar resultados estéticos pobres. En vez del colgajo de transposición de Fricke, otra alternativa para el cierre del sitio donante es el uso de colgajos bipediculados $5,34,47,49$.

Colgajo de Tulip: es un colgajo axial bilobulado, que incluye la arteria temporal superficial en la frente y que debe trasladarse como un colgajo en isla hasta el canto externo. En algunos casos, cada lóbulo del colgajo puede tener un suministro sanguíneo axial independiente, ya que se encuentran variaciones anatómicas de las ramas de la arteria temporal superficial que se los provee. Para el diseño del colgajo puede practicarse una ecografía Doppler, que ayuda a localizar fácilmente la arteria temporal superficial para asegurarse de que el suministro sanguíneo esté incorporado ${ }^{5,34,50}$.

Reparación del tendón del canto lateral: se requiere cuando se interrumpe la continuidad entre el tubérculo de Whitnall y el tendón del canto lateral, en la región más interna de la órbita lateral. Cuando se emplea el periostio de la órbita para soportar el canto lateral, se describe que éste ejerce un vector con dirección superolateral, aunque no siempre es suficiente. Para suturar el tubérculo de Whitnall, el periostio puede transponerse como un colgajo ${ }^{5,47,49}$.

Para fijar el tendón al hueso, se han descrito tres técnicas que se utilizan con cierta frecuencia. Una de ellas consiste en perforar un orificio a través de la órbita lateral, a $2 \mathrm{~mm}$ del borde orbitario en la región del tubérculo de Whitnall, y pasar un alambre de titanio de calibre 26 o 28 que se cierra a sí mismo formando un aro, para fijar la sutura del tendón a la órbita ósea. Sin embargo, esta técnica puede dejar el alambre palpable y producir artefactos en las imágenes radiológicas, por lo que puede optarse por el uso de suturas de anclaje 
óseo emparejadas que se pasan a través del orificio y se ligan en la parte externa. Bachelor describió una técnica usando un colgajo en forma de Y tomado del tendón del palmar largo, que se lleva y asegura mediante un orificio en la órbita lateral, para reconstruir el canto lateral cuando el déficit es tan extenso que compromete las placas tarsales superior e inferior ${ }^{5,47,49,50}$.

Defectos de espesor completo: cuando se pierde todo el espesor de la piel en el canto lateral, suele también perderse estructuras adyacentes como los párpados. Jones y Cooper reconocieron que la reconstrucción de estos defectos requería tanto de la reconstrucción del canto lateral, como de la de los párpados y platillos tarsales. Para reemplazar estas estructuras, se cuenta con materiales autógenos, como injertos o colgajos tarsales, mucosa del paladar duro y tendón del palmar largo, entre otros, además de esclerótica de cadáver, fascia o piel artificial, de animal o de cadáver. En otros casos, para la reconstrucción de defectos muy complejos del canto lateral, puede requerirse una combinación de varias técnicas 5,34,50.

Una complicación importante se presenta cuando, durante la resección tumoral o la reconstrucción del canto externo, se recorta en exceso el párpados superior o el inferior (más común), lo cual lleva a una mala posición del canto lateral, con pobre adhesión del párpado si es coloca muy anterior, o a triquiasis e irritación de la superficie ocular si queda muy posterior. La mala posición hacia arriba o hacia abajo (más común) puede dar un pobre resultado estético $0^{5,34,50,51}$.

\section{Complicaciones posoperatorias y seguimiento}

Entre las principales complicaciones de estas cirugías de reconstrucción que implican la región periorbitaria, se pueden mencionar retracción de la mejilla o del párpado, ectropión cicatricial, lagoftalmos y ptosis palpebral. Según la experiencia de Kikkawa, la incidencia aproximada de una mala posición palpebral sintomática que requiere de una segunda intervención, es de $10 \%$.

\section{Imbricación palpebral}

Es una complicación frecuente durante la cantotomía lateral con cantólisis o sin ella, que consiste en una asimetría de los planos de reparación de los tendones superior e inferior del canto, por un error en la identificación y aproximación de los tendones del canto y de los márgenes palpebrales. Esto produce un defecto estético, además de un defecto funcional, por la superposición de un párpado sobre el otro a nivel del canto lateral. El manejo de esta complicación es liberar los tendones del canto en la correcta posición anatómica y volver a suturar por planos, ${ }^{5,51}$.

\section{Ectropión}

Consiste en la eversión del borde libre del párpado inferior y puede resultar por un inadecuado soporte del tarso, del tendón del canto lateral o de ambos. Por la edad, y la laxitud de la piel y del tendón del canto lateral subyacente, puede presentarse un ectropión involutivo, que resulta en la exposición de la esclerótica inferior. El recortar en exceso la lamela anterior (piel y músculo) del párpado inferior, puede causar su contractura, lo que resulta en un ectropión cicatricial. Para un ectropión menor, una buena opción de corrección es el masaje de la cicatriz queloide para corregir la retracción del párpado. Sin embargo, en el ectropión más pronunciado, una nueva intervención quirúrgica con ablación de la cicatriz y aumento del tejido lamelar (por reordenamiento del tejido o del injerto) puede ser la única solución para prevenir problemas mayores por la exposición ocular ${ }^{5,51}$.

\section{Entropión}

Es la inversión del margen palpebral, que puede llevar a complicaciones mayores por excoriaciones corneales, con subsecuente dolor, irritación ocular, infecciones, cicatrices y disminución visual, producidas por la inversión de las pestañas que se dirigen hacia la córnea. Las estrategias de manejo para el entropión son paralelas a las de ectropión, y pueden enfocarse en reemplazar los defectos de la lámina posterior con injertos o colgajos autógenos, animales, cadavéricos, artificiales, fibrosos, mucosos o fibrosos y mucosos (por ejemplo, tarso, paladar duro, cartílago auricular y esclerótica) $)^{5,51}$.

El seguimiento puede variar dependiendo del caso. Por lo general, es mejor revisar a los pacientes después de 24 horas, a la semana, a los 15 días y al mes. En estos controles se deben que tener en cuenta las posibles complicaciones, retirar el capitoneo de los injertos, desbridar y drenar si es necesario. Posteriormente, se hacen controles cada seis meses en los primeros dos años y luego un control anual durante los primeros 10 años. Además, en casos de tumores más profundos y, especialmente, en aquellos con invasión perineural, se recomienda hacer un seguimiento estricto, al menos, cada seis meses durante cinco años, para descartar recurrencias ${ }^{9,13}$.

\section{Conclusiones}

Actualmente la cirugía micrográfica de Mohs es la opción terapéutica y diagnóstica más completa y con menores tasas de recidivas para el tratamiento quirúrgico 
de tumores cutáneos complejos y agresivos localmente, además de que es la única alternativa que permite ahorrar tejido sano con la seguridad de erradicar el tumor.

La reconstrucción inmediata que se hace el mismo día de la erradicación tumoral, brinda menor tasa de retracción e infección del área del defecto y proporciona comodidades para nuestros pacientes y familiares. Además, permite ahorrar en costos ya que no toca hacer una segunda cirugía. Sin embargo, este tipo de estudios no permiten determinar la efectividad ni la eficacia del tratamiento.

Cuando el defecto posee un lecho con adecuado soporte vascular, los injertos son viables, en caso contrario, podría ser necesario el uso de colgajos.

Los resultados quirúrgicos dependen de muchos factores, como son el área tratada, la dimensión del defecto por cubrir y la edad de los pacientes. El éxito de la reconstrucción quirúrgica después de la resección de tumores, se refleja, no solo en un buen resultado estético, sino también en la conservación de la funcionalidad. Sin embargo, no existe consenso sobre cuál es la mejor técnica quirúrgica. La mejor puede ser con la que el cirujano se encuentre más familiarizado, ya que entre mayor sea su experiencia y el número de procedimientos del mismo tipo que haya practicado, mejores son los resultados estéticos y funcionales.

La inadecuada reconstrucción del área periorbitaria puede llevar a complicaciones más serias, como el ectropión o el entropión, que finalmente pueden causar daños de la córnea. Se sugiere realizar estudios analíticos para establecer una mejor 'evidencia', teniendo en cuenta que el tema es de frecuente manejo por los cirujanos dermatólogos en nuestro país.

\section{Conflicto de intereses}

Este artículo fue elaborado exclusivamente por los autores y apoyado por la Fundación Universitaria de Ciencias de la Salud, sin ningún tipo de patrocinio de entidades externas.

\section{Referencias}

1. Kikkawa D, Boddy S, Christine C. Reconstruction of large and complex periorbital defects from cutaneous cancer. International Ophtalmology Clinics. 2009;49:237-45.

2. Kummoona R. Periorbital and orbital malignancies: Methods of management and reconstruction in Iraq. J Craniofac Surg. 2007;18:1370-5.

3. Actis A, Actis G. Reconstruction of the upper eyelid with flaps and free grafts after excision of basal cell carcinoma. Case Rep Ophthalmol. 2011;2:347-53.
4. Moul CP, Peggy L, Peter R, Shumaker P, Daniel E, Hugh T. Mohs micrographic surgery for eyelid and periorbital skin cancer. International Ophthalmology Clinics. 2010;49:1536-9617.

5. Brian T, Lin J, Rubin P. Lateral canthal reconstruction after headneck or periocular cutaneous malignancy. International Ophthalmology Clinics. 2009;49:195-206.

6. Arquero P. Tratamiento reparador y reconstructor de las secuelas de la patología quirúrgica facial, 1998-2011. Fecha de consulta: Octubre-2012 Disponible en: http://www.clinicaarquero.com/10 propedeutica10.htm.

7. Baker AI, Beas CK, Bloom BD. Colgajos locales en la reconstrucción facial. Philadelphia, editorial Mosby-Elsevier; 2010. Pág. 786

8. Cook B, Bartley G. Treatment options and future prospects for the management of eyelid malignancies. An Evidence-Based Update Ophthalmology. 2001;108:2088-98.

9. Mohs FE. Micrographic surgery for the microscopically controlled excision of eyelid cancers. Arch Ophthalmol. 1986;104:901-9.

10. Bernardini F. Management Of malignant and benign eyelid lesions. Curr Opin Ophthalmol. 2006;17:480-4.

11. Gilberg S, Tse D. Malignant eyelid tumors. Ophthalmol Clin North Am. 1992;5:261-85.

12. Khachemoune A, Suzanne M, Johnson D. Microcystic adnexal carcinoma: Report of four cases treated with Mohs' micrographic surgical technique. Int J Dermatol. 2004;44:507-12.

13. Lindgren G, Lindblom B, Larko O. Mohs' micrographic surgery for basal cell carcinomas on the eyelids and medial canthal area. Reconstruction and follow-up. Acta Ophthalmol Scand. 2000;78:1395-3907.

14. National Comprehensive Cancer Network. Guidelines index basal cell and squamous cell TOC staging, MS, Clinical Practice Guidelines in Oncology. Washigton. Clinical Practice Guidelines Ed.; 2012. Pág. 1-22.

15. Zaragosa P, Menéndez F, Gómez T. Carcinoma basocelular palpebral. Madrid, España, 2006. Fecha de consulta: Septiembre-2012. Disponible en: http://www.oftalmo.com/studium/studium2006/ studo6-4/06d-03.htm.

16. Doxanas M, Green W, Iliff E. Factors in successful surgical management of basal cell carcinoma of the eyelids. Am J Ophthalmol. 1981;91:726-36.

17. Maloof A, Leatherbarrowe B. The maximal Hughes procedure. Ophtal Plast Reconstr Surg. 2001;17:96-102.

18. Margo E, Waltz K. Basal cell carcinoma of the eyelid and periocular skin. Surv Ophthalmol. 1993;38:169-92.

19. Wong V, Marshall A, Whitehead K. Management of periocular basal cell carcinoma with modified en face frozen section controlled excision. Ophthal Plast Reconstr Surg. 2002;18:430-5.

20. Reifler DM, Hornblass A. Squamous cell carcinoma of the eyelid. Surv Ophthalmol. 2004;30:349-65.

21. Leibovitch I, Huilgol C, Richards S. periorbital microcystic adnexal carcinoma: Management and outcome with Mohs Micrographic surgery. Ophthalmologica. 2006;220:109-13.

22. Nemet AY, Deckel Y, Martin PA. Management of periorbital basal and squamous cell carcinoma: A series of 485 cases. Am J Ophthalmol. 2006;142:293-7.

23. Peters GB MD, Shields JA. Management and prognosis of merkel cell carcinoma of the eyelid. Ophthalmology. 2001;108:1575-9. 
24. Inkster C, Ashworth J, Murdoch JR. Oculoplastic reconstruction following Mohs surgery. Eye. 1998;12:214-8.

25. Kersten RC, Ewing-Chow D, Kulwin DR, Gallon M. Accuracy of clinical diagnosis of cutaneous eyelid lesions. Ophthalmology. 1997;104:479-84.

26. Lai M, Cheney ML. Temporoparietal fascial flap in orbital reconstruction. Arch Facial Plast Surg. 2000;2:196-201.

27. Weng C. Periorbital soft-tissue and socket reconstruction. Ann Plast Surg. 1995;34:457-62.

28. Bowman PH, Fosko SW, Hartstein ME. Periocular reconstruction. Semin Cutan Med Surg. 2003;22:263-72.

29. Harris GJ, Pérez N. Anchored flaps in post-Mohs reconstruction of the lower eyelid, cheek, and lateral canthus: avoiding eyelid distortion. Ophthal Plast Reconstr Surg. 2003;19:5-13.

30. Patrinely JR, Marines HM, Anderson RL. Skin flaps in periorbital reconstruction. Surv Ophthalmol. 1987; 31 (4):249-61.

31. Kaufman AJ. Periorbital reconstruction with adjacent-tissue skin grafts. Dermatol Surg. 2005;31:1704-6.

32. Matsumoto K, Nakanishi H, Urano Y. Lower eyelid reconstruction with a cheek flap supported by fascia lata. Plast Reconstr Surg. 1999;103:1650-4.

33. Khan JA, Garden VS. Combined flap repair of moderate lower eyelid defects. Ophthal Plast Reconstr Surg. 2002;18:202-4.

34. Codner MA, Wolfli JN, Anzarut A. Primary transcutaneous lower blepharoplasty with routine lateral canthal support: A comprehensive 10-year review. Plast Reconstr Surg. 2008;121:241-50.

35. Zarem H, Resnick J. Expanded applications for transconjunctival lower lid blepharoplasty. Plast Reconstr Surg. 1999;88:215-20.

36. Patipa $M$. The evaluation and management of lower eyelid retraction following cosmetic surgery. Plast Reconstr Surg.106:479-84.

37. Yanaga $\mathrm{H}$, Mori S. Eyelids and eye socket reconstruction using the expanded forehead flap and scapha composite grafting. Plast Reconst Surg. 2001;108:8-16.

38. Scuderi N, Rubino C. Island chondro-mucosal flap and skin graft: A new technique in eyelid reconstruction. Br J Plast Surg. 1994;47:57-9.
39. Porfiris E, Georgiou P, Harkiolakis G. Island mucochondrocutaneous flap for reconstruction of total loss of the lower eyelid. Plast Reconstr Surg. 1997; 100 (1):104-7.

40. Lagares A, De lope F, Góngora J, Barrera F, Gómez T. Reconstrucción de párpado inferior mediante colgajos miocutáneos en isla de los músculos orbicular y nasal. Cir Plást Iberolatinoam. 2009;35:35-42.

41. Callahan MA, Callahan A. Mustarde flap lower lid reconstruction after malignancy. Ophthalmology. 1980;87:279-86.

42. Shields JA, Demirci H MB. Sebaceous carcinoma of the eyelids: Personal experience with 60 cases. Ophthalmology. 2004;111:2151-7.

43. Harris G, Logani SC. Multiple aesthetic unit flaps for medial canthal reconstruction. Ophthal Plast Reconstr Surg. 1998;14:352-9.

44. Jelks GW, Glat P, Jelks EB, Longaker M. Medial canthal reconstruction using a medially based upper eyelid myocutaneous flap. Plast Recontr Surg. 2002;110:1636-43.

45. Suárez R, Valdivieso M, Martínez D, Mauleón C, Colgajos cutáneos -los más usados. Cirugía Dermatológica. 2001;4:319-25.

46. Vayvada H, Menderes A, Tan Ö, Yilmaz M. Total lower eyelid reconstruction using paranasal flap. J Craniofac Surg. 2006;17(5):1020-6.

47. Jones IS, Cooper WC. Lateral canthal reconstruction. Trans Am Ophthalmol Soc. 1973;71:296-302.

48. Jelks G, Glat P, Jelks B, Longaker T. The inferior retinacular lateral canthoplasty: A new technique. Plast Reconstr Surg. 1997;100:1262-70.

49. Glat P, Jelks G. Evolution of lateral canthoplasty: Techniques and indications. Plast Reconstr Surg. 1997;100:1396-414.

50. Cologlu H, Kocer U, Oruc M. Axial bilobed superficial temporal artery island flap (tulip flap): Reconstruction of combined defects of the lateral canthus including the lower and upper eyelids. Plast Reconstr Surg. 2007;119:2080-7.

51. Leone CR. Lateral canthal reconstruction. Ophthalmology. 1987;94:238-41. 